\title{
Une nouvelle imagerie ostéo-articulaire basse dose en position debout : le système EOS
}

\author{
J. DUBOUSSET ${ }^{1}$, G. CHARPAK ${ }^{2}$, I. DORION ${ }^{2}$, W. SKALLI ${ }^{3}$, F. LAVASTE ${ }^{3}$, \\ J. DEGUISE ${ }^{4}$, G. KALIFA ${ }^{5}$, S. FEREY ${ }^{5}$
}

(Manuscrit reçu le 10 février 2005, accepté le 21 février 2005)

RÉSUMÉ L'association et la collaboration étroite de plusieurs disciplines (physique des rayonnements, biomécanique, radiologie et orthopédie de l'enfant) a permis la mise au point dans notre pays et le développement d'un nouvel appareil d'imagerie dénommé EOS dont les principales caractéristiques sont: (1) la réduction considérable des doses de rayons $X$ (de 8 à 10 fois moins pour la radiologie bidimensionnelle, de 800 à 1000 fois moins pour la tomodensitométrie tridimensionnelle) grâce au détecteur gazeux inventé par Georges Charpak qui lui ont valu le prix Nobel. (2) L'étude du patient en position debout obtenant des clichés simultanés de face et de profil du sommet de la tête jusqu'à la plante des pieds. (3) La possibilité de reconstruction 3D de tous les niveaux ostéo-articulaires a été vérifiée aussi précise que celle obtenue par tomodensitométrie conventionnelle. De plus, l'examen est effectué en position fonctionnelle debout ou assise, ce qui n'était pas possible avec les appareils de tomodensitométrie actuellement tous en position couchée. La reconstruction 3D peut être obtenue dans des délais acceptables de 15 à 30 minutes pour un rachis complet. Ne faisant pas double emploi avec l'IRM, même si celle-ci évolue aussi vers la position debout, EOS permettra des études de la pathologie ostéo-articulaire jusque-là jamais réalisées (en particulier du rachis et des membres inférieurs) avec un examen d'ensemble de l'individu au lieu des segments fragmentés donnés jusqu'à présent par les moyens actuels, radiographies conventionnelles ou tomodensitométrie.

ABSTRACT A new imaging 2D and 3D for musculo-skeletal physiology and pathology with low radiation dose and standing position: the EOS system.

Very precise combined work between multidisciplinary partners (radiation engineers in physics, engineers in biomecanics, medical radiologists and orthopedic pediatric surgeons) lead to the concept and development of a new low dose radiation device named EOS. This device allows 3 main advantages: (1) thanks to the invention of Georges Charpak (Nobel Price 1992) who designed gaseous detectors for X-rays, the reduction of dose necessary to obtain a good image of skeletal system was 8 to 10 times less for 2D imaging, compared to the dose necessary to obtain a 3D reconstruction from CT scan cuts the reduction factor was 800 to 1000 . (2) The

\footnotetext{
Membre de l'Académie nationale de médecine et hôpital St. Vincent de Paul, Service de chirurgie orthopédique, Paris, France.

Biospace, Instruments, Paris, France.

École nationale supérieure des arts et métiers, Paris, France.

Laboratoire de recherche en imagerie orthopédique, Montréal, Canada.

Hôpital St. Vincent de Paul, Service de radiologie, Paris, France.
} 


\begin{abstract}
accuracy of 3D reconstruction obtained is as good as a 3D reconstruction from CT scan cuts. (3) The patient in addition get its imaging in standing functional position thank to the X-rays obtained from head to feet simultaneously AP and lateral. This is a big advantage compared to $\mathrm{CT}$ scan used only in lying position. From this simultaneous AP and lateral X-rays of the whole body thanks to the 3D bone external envelop technique, the engineers in biomechanics allowed to obtain 3D reconstruction of every level of osteo-articular system in standing position with an acceptable period of time (15 to 30 minutes). This (in spite of the evolution of standing MRI) allows more precise bone reconstruction in orthopedics especially at the level of spine, lower limbs, etc. In addition the fact to study the entire skeleton in standing functional position instead of small segmented studies given by CT scan in lying position produce a real improvement as well for physiology as for pathology of bone and joints disorders and especially for spinal pathology.
\end{abstract}

Key words: Radiography / 3D reconstruction / Standing position

\title{
1. Introduction
}

L'invention de Georges Charpak (prix Nobel de physique 1992) consistant à obtenir des radiographies en économisant considérablement les doses des radiations nécessaires à l'obtention d'une bonne image grâce aux détecteurs gazeux, a conduit une équipe multidisciplinaire comprenant ingénieurs physiciens, ingénieurs biomécaniciens et cliniciens radiologues et orthopédistes à mettre au point et utiliser un nouvel appareil d'imagerie médicale dénommé EOS.

Cet appareil comporte une double innovation, puisqu'il permet d'obtenir des images en 2D, mais aussi en 3D en position debout de l'ensemble du squelette de la tête aux pieds, et ceci grâce à des nouvelles techniques de reconstruction 3D développées conjointement entre le Laboratoire de biomécanique de l'ENSAM (École nationale des arts et métiers) à Paris LBM-CNRS UMR 8005 (Laboratoire de biomécanique associé au Centre national de la recherche scientifique) à Paris et le Laboratoire de recherches en imagerie et orthopédique (LIO) à Montréal (Deschenes et al., 2003).

Il en découle de multiples applications, aussi bien en physiologie qu'en pathologie ostéo-articulaire qui amène un nouveau champ d'investigations et d'applications. L'avantage majeur du système est la diminution considérable des doses de Rx dont on connaît les risques (Berrington de Gonzales et Darby, 2004) allant d'un facteur de réduction 10 pour les radios conventionnelles $2 \mathrm{D}$ à un facteur de 800 à 1000 pour les reconstructions 3D obtenues jusqu'alors grâce aux tomodensitométries qui avaient en outre l'inconvénient de ne pouvoir être utilisées qu'en position couchée. 


\section{Bases physiques - Les premiers appareils et l'évolution jusqu'au modèle actuel}

En 1996, Georges Charpak et la société Biospace Instruments ont mené une première évaluation de l'intérêt des inventions de Georges Charpak dans le domaine de la radiologie médicale. Les détecteurs gazeux qui lui ont valu le prix Nobel de physique (1992) permettent en effet la conversion, dans un gaz sous pression tel que le xénon, de photons $\mathrm{X}$ en électrons. Ces électrons sont amplifiés par effet d'avalanche, c'est-à-dire une multiplication du nombre des électrons dans le champ électrique et détectés par une chaîne électronique adaptée (Babichev et al., 2003).

Un prototype conçu avec une technologie de détecteurs gazeux de première génération a été installé au CHU St. Vincent de Paul, dans le service de radiologie pédiatrique du Professeur Kalifa. Plus de 1000 examens radiologiques ont été réalisés. L'évaluation clinique a été menée sous contrôle du CEDIT (Centre d'évaluation des innovations technologiques) pendant 18 mois, sur un échantillon de 200 enfants et adolescents, dans le cadre d'un protocole soumis au CCPPRB (Comité consultatif de la protection des personnes lors des recherches biologiques) de Cochin-Port Royal portant sur la dosimétrie, la qualité d'image et l'évaluation clinique de clichés pulmonaires, du bassin et du rachis. Les mesures de dosimétrie ont été effectuées par le CAATS (Centre d'assurance de qualité des applications technologiques dans le domaine de la santé), organisme indépendant accrédité par la Communauté européenne pour l'établissement des recommandations relatives à la dosimétrie. Les facteurs de réduction de doses mesurées lors de ces essais par comparaison aux films classiques étaient de 4 à 20, selon les localisations (Kalifa et al., 1998).

L'analyse des critères diagnostiques et de qualité a conduit en 1997 à des conclusions très positives en terme de réduction de doses et plus réservées en terme de qualité d'image révélant essentiellement les difficultés d'interprétation par les lecteurs de ces images, d'autant plus nouvelles que peu de services de radiologie étaient à l'époque équipés d'imagerie digitale.

Certains handicaps de l'appareil testé ont également été soulignés à l'époque. Les faiblesses constatées de cette première génération d'équipements concernaient essentiellement :

- la résolution encore faible $(1 \mathrm{~mm})$,

- la lenteur de la prise d'image (8 à 10 secondes pour un rachis).

De nouvelles technologies de détecteurs utilisant les circuits imprimés de haute densité et des modifications de l'architecture interne des détecteurs ont depuis 
ouvert la voie à des résolutions de l'ordre de $250 \mu \mathrm{m}$ parfaitement adaptées à l'imagerie de l'os. Ces détecteurs permettent en outre d'obtenir des images de très grande dynamique, ou profondeur, c'est-à-dire possédant un nombre de niveaux de gris discernables très important : 30 à 50 000, contre de l'ordre d'une centaine de niveaux avec le film classique. L'affichage sur un écran digital permet de sélectionner la gamme de niveaux de gris d'intérêt pour la lecture. Ces détecteurs sont enfin insensibles au rayonnement diffusé et sensibles à un photon $\mathrm{X}$ unique : ils permettent donc l'acquisition d'images de grande qualité radiologique, au prix d'une irradiation patient très faible.

En parallèle, les équipes du LBM et du LIO ont développé ensemble de nouvelles méthodes de reconstruction tridimensionnelle précise de vertèbres, puis progressivement des autres éléments du squelette, à partir de seulement deux radiographies prises sous deux incidences. La méthode de reconstruction consiste à déterminer la forme et la position spatiale de l'élément d'intérêt (vertèbre, fémur, bassin, ...), en cohérence avec les informations repérables sur les radiographies (contours en particulier).

La démarche générale consiste à positionner approximativement dans l'espace un élément de forme proche (appelé élément générique). Cet élément permet de déterminer des contours radiographiques virtuels. La forme et la position sont alors modifiés jusqu'à ce que les contours virtuels se superposent au mieux avec les contours réels relevés sur la radiographie.

Georges Charpak et la société Biospace Instruments ont ainsi lancé en 2000 le développement d'un instrument de radiologie utilisant ces nouvelles technologies dans le contexte particulier de l'orthopédie, pour lequel une capacité d'imagerie tête aux pieds, en position debout, est particulièrement nécessaire. L'appareil comprend un détecteur linéaire de largeur $45 \mathrm{~cm}$ balayé verticalement en 10 à 20 secondes pour assurer une couverture totale de $170 \mathrm{~cm}$ (en hauteur) par $45 \mathrm{~cm}$ (en largeur), ouvrant ainsi la voie à une analyse radiographique globale du patient. Deux ensembles orthogonaux composé chacun d'une source de rayon $\mathrm{X}$ et d'un détecteur, unis physiquement, permettent la prise de vue simultanée des clichés face et profil quasi systématiquement requis en orthopédie.

L'appareil EOS, opérationnel depuis 2003, permet ainsi la prise de deux clichés tête aux pieds, digitaux, à faible irradiation. Ces clichés sont utilisables tant pour l'examen de zones anatomiques peu denses (zone cervicale par exemple) que pour l'examen de zones très atténuantes (zone lombaire par exemple) grâce à la très grande performance du détecteur en termes de dynamique. 


\section{La validation radiographique et la dosimétrie}

Il était bien sûr très important de valider la qualité des radiographies obtenues avec EOS, mais aussi la dosimétrie mesurée à la fois pour les clichés $2 \mathrm{D}$ et les reconstructions $3 \mathrm{D}$, de manière à les comparer aux appareils d'imagerie conventionnelle radiographique et de tomodensitométrie.

Avec l'agrément du comité d'éthique du CCPPRB (Comité consultatif pour la protection des personnes lors des recherches biologiques) de l'hôpital Cochin, l'étude a été menée sur un examen de routine de radiologie pédiatrique, à savoir le rachis entier des malades scoliotiques. Cet examen a été choisi en raison de l'importance des doses délivrées pour ces clichés de face et de profil, en particulier au niveau des organes sensibles (mœlle osseuse, seins, gonades) mais aussi en raison de la fréquence de ces examens souvent nécessaire, de manière répétitive pour vérifier l'évolutivité avec le temps de ces déformations et leur contrôle après traitement orthopédique ou chirurgical.

L'étude visait la mesure de la réduction de dose et la qualité de l'image obtenue, ainsi que celle des résultats des reconstructions 3D de surface pouvant aussi être obtenue par TDM.

45 enfants ont été étudiés et les résultats obtenus suivent.

L'irradiation pour les examens classiques face et profil du rachis étaient réduits de 8 à 10 fois par rapport à la radiologie conventionnelle actuelle, comme en témoigne le tableau I, au niveau de la peau par exemple.

TABLEAU I

Paramètres radiographiques utilisés et dosimétrie au niveau de la peau. Radiographic parameters used and dosimetry on the skin.

\begin{tabular}{|c|c|c|c|c|c|}
\hline & \multicolumn{2}{|c|}{$\mathrm{KVp}$} & \multicolumn{2}{|c|}{ Dose de peau ( $\mu \mathrm{Gy})$} & \multirow{2}{*}{ Facteur de réduction } \\
\hline & Film & EOS & Film & EOS & \\
\hline $\begin{array}{c}\text { Face } \\
\text { (moyenne) }\end{array}$ & 71 & 69 & 1196 & 127 & 9,4 \\
\hline $\begin{array}{c}\text { Profil } \\
\text { (moyen) }\end{array}$ & 83 & 84 & 1618 & 192 & 8,4 \\
\hline
\end{tabular}

La comparaison EOS / TDM avec reconstruction tridimensionnelle montre que la diminution de la dose absorbée va d'un facteur 100 pour les organes génitaux à plus de 600 pour la thyrö̈de. 


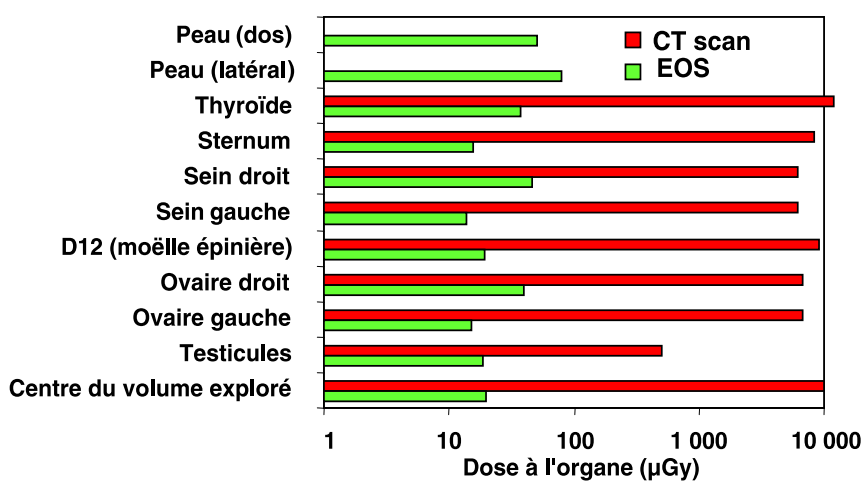

Figure 1.

La figure 1 est particulièrement explicite.

\section{Reconstruction tridimensionnelle}

Les données de base de la reconstruction tridimensionnelle se rapportent (Laporte et al., 2003; Le Bras et al., 2003; Mitton et al., 2003; Pomero et al., 2004). Aux images radiographiques calibrées. Dans le système EOS, du fait de la liaison physique entre les ensembles sources détecteurs, la position dans l'espace des capteurs et des sources de rayons X est parfaitement connue : l'environnement radiographique est donc pré-calibré.

La projection de la pièce osseuse radiographiée est comparée à celle d'un objet virtuel dit objet générique qui en a approximativement la forme. Cet objet est défini par les coordonnées d'un nuage de points répartis à sa surface (400 à 9000 points en fonction de la complexité de l'objet). Il peut être obtenu à partir d'une pièce anatomique d'une reconstruction TDM 3D ou d'un modèle statistique.

Les informations extraites de l'image radiographique permettent d'identifier des repères ponctuels (par exemple centre géométrique de la tête fémorale), ou des contours (par exemple contours associés à la projection des condyles fémoraux). Certains repères sont identifiables simultanément sur les deux images radiographiques (stéréo-correspondants), et sont associés à un repère anatomique spécifique, d'autres peuvent seulement être associés à une région, la localisation précise dans la région dépendant de l'orientation de l'objet.

Les méthodes de reconstruction sont spécifiques aux objets reconstruits et à l'image radiographique de ces objets. Deux exemples illustreront les démarches possibles. 
La reconstruction d'une extrémité distale de fémur: elle commence par le repérage des contours de l'os sur les images radiographiques dans les deux plans orthogonaux. Ces contours ne sont pas nécessairement stéréo correspondants. Ils sont ensuite comparés à ceux d'un fémur virtuel projeté sur ces mêmes images. Le fémur virtuel va être déformé, d'abord par une succession de translations, rotations, homothéties, puis par une déformation non linéaire jusqu'à obtenir une superposition des contours réels et virtuels.

La reconstruction des vertèbres d'un rachis scoliotique : on commence par pointer semi automatiquement quatre repères anatomiques par corps vertébral, sur les images radiographiques des deux plans orthogonaux. Ceci permet de quantifier pour chaque corps vertébral, son volume de confinement dans l'espace, l'inclinaison latérale et sagittale de la vertèbre et de définir la ligne vertébrale qui joint les centres des corps vertébraux. De plus, la rotation vertébrale axiale est estimée à partir des connaissances anatomopathologiques des courbures rachidiennes. Un modèle théorique issu de mesures directes sur 1628 vertèbres sèches et de reconstruction 3D de 96 rachis est alors construit, puis rétro-projeté sur l'image radiographique. Semi automatiquement on réoriente l'objet virtuel pour corriger la rotation vertébrale axiale et on rectifie la position des repères rétroprojetés jusqu'à obtenir une bonne adéquation entre les contours réels et les contours virtuels de l'objet modélisé.

L'automatisation complète de ce processus est en cours.

\section{Validation de la reconstruction $3 D$}

La validation des reconstructions a été effectuée pour des vertèbres, des fémurs et des tibias, d'abord in vitro sur pièces sèches, puis in vivo. Dans chaque cas les reconstructions 3D sont effectuées d'une part à partir de coupes TDM millimétriques ( $2 \mathrm{~mm}$ pour des vertèbres scoliotiques), et d'autre part à partir de la simple paire de radiographies fournie par EOS. Chaque reconstruction conduit à la définition d'un nuage de points décrivant la surface enveloppe de l'os considéré (500 à 2000 points par os). Une superposition de ces deux nuages de points permet d'estimer les erreurs, la TDM étant considérée comme la référence. In vitro, pour 36 vertèbres, 25 fémurs proximaux, 25 fémurs distaux et 7 tibias, l'erreur moyenne est de $0,9 \mathrm{~mm}$, et $95 \%$ des erreurs sont inférieures à $2,4 \mathrm{~mm}$. In vivo, et même dans le cas d'os très déformés (scoliose sévère et genoux arthrosiques), l'erreur moyenne reste inférieure à $1,5 \mathrm{~mm}$.

\section{Conséquences}

On constate donc la réduction considérable des doses d'irradiation qui peut être obtenue avec le système EOS associé aux méthodes informatiques de 
reconstruction 3D obtenue à partir des simples radiographies face et profil simultanées dont la validation de la précision surfacique comparée à celle de la TDM s'est révélée tout à fait identique.

Quant à la qualité des images obtenues, elle est tout à fait comparable à celle d'une radiographie classique pour, non seulement l'examen de la colonne vertébrale, mais aussi simultanément celui du bassin, du thorax, et des membres inférieurs par exemple.

La possibilité de jouer sur le fenêtrage des images a pour EOS les mêmes caractéristiques que celles de la radiographie numérisée évitant une nouvelle irradiation (nécessaire en cas d'erreur technique) comme on le voit dans la radiographie conventionnelle.

L'analyse des tissus de contraste différents (os, muscles, poumons, ligaments) sera peut-être une autre possibilité en cours de validation avec EOS.

\section{Applications pratiques existantes et potentielles}

Les premières applications pratiques sont les mêmes indications que celles de la radiographie conventionnelle pour ce qui est du système squelettique avec trois avantages majeurs :

- la diminution des doses d'irradiation (de 8 à 10 fois inférieures) ;

- la non nécessité de refaire des clichés lorsqu'on ne visualise pas bien une région, car on peut jouer grâce au numérique sur les valeurs de gris et faire apparaître l'image avec une précision largement suffisante au niveau intéressé, comme on peut l'avoir avec des radiographies numériques actuelles ;

- l'étude se fait en station debout tout à fait fonctionnelle, pour le squelette entier des pieds à la tête et en particulier par rapport aux imageries en coupes (TDM) actuelles qui sont réalisées en position couchée.

Donc tout à fait intéressant en remplacement des examens radiologiques répétitifs et/ou irradiants : bilans répétitifs des affections rachidiennes opérées ou non chez l'enfant, l'adolescent ou l'adulte, bilans squelettiques du bassin et des membres inférieurs chez l'enfant, clichés thoraciques de dépistage.

Les secondes applications pratiques découlent de la possibilité d'obtenir des reconstruction tridimensionnelles aussi fiables que celles obtenues avec la tomodensitométrie avec les deux avantages majeurs :

- la diminution considérable des doses d'irradiation (de 800 à 1000 fois inférieures) ;

- la réalisation de l'examen en position debout ou assise donc fonctionnelle.

Ces éléments sont d'importance majeure pour : la pathologie rachidienne. 
L'exploration globale du corps permet de déceler et de mesurer les compensations qui se produisent au niveau non seulement du rachis, mais de l'ensemble du corps dans l'équilibre et la fonction de l'individu. Une plate forme de force peut être intégrée au système EOS, permettant de connaître la ligne de gravité passant par le centre du polygone de sustentation, et de la rapporter aux données précédentes. La modélisation de l'enveloppe cutanée commence à être réalisée. Les principales indications rachidiennes sont à l'heure actuelle :

- le suivi évolutif des affections rachidiennes au cours de la croissance et du vieillissement du rachis ;

- l'établissement des bilans pré-opératoires, en tenant compte des éléments extra rachidiens (tête, bassin, hanches, genoux), tout autant que du rachis lui-même, en particulier pour le plan horizontal difficile à appréhender sur les radiographies conventionnelles et qui est parfaitement démontré par la reconstruction volumétrique 3D, par exemple vu d'en haut ;

- la surveillance post-opératoire, notamment de l'équilibre global et des compensations immédiates ou à long terme ;

- le contrôle des actions des corsets orthopédiques. Ceci grâce à la reconstruction thoracique qui permet de visualiser dans l'espace la position des appuis et leurs conséquences, de même que l'effet sur le bassin et l'ensemble rachidien depuis la vertèbre céphalique jusqu'à la vertèbre pelvienne ;

- la mesure de la capacité thoracique et du volume thoracique pouvant permettre grâce à cette mesure simple et peu irradiante d'éviter certains examens par TDM thoracique à coupes jointes, beaucoup plus irradiantes pour l'évaluation des volumes pulmonaires au cours de la croissance et détecter les effets favorables ou néfastes des divers appareillages ;

- l'estimation et la mesure de la déformation rachidienne de façon beaucoup plus réaliste que les mesures et pourcentages de correction donnés par le simple angle de Cobb rachidien qui en réalité ne mesure que l'effondrement rachidien projeté dans un seul plan.

Cet ensemble de regards nouveaux sur la pathologie rachidienne amènera à n'en pas douter une évolution dans les indications opératoires et même probablement dans les techniques de correction.

Il faut enfin signaler que l'association EOS et mesures spatiales 3D réalisée avec des instruments non invasifs (ultrasons ou palpateurs externes) permet d'avoir une idée assez précise de la mobilité relative à la ligne de gravité élevée à partir du polygone de sustentation, la superposition de cette ligne de gravité de référence rendant l'utilisation des 2 types d'appareils tout à fait valable et complémentaire. 


\section{Le début des études sur la morphologie tridimensionnelle en position fonctionnelle debout des divers secteurs et articulations des membres inférieurs est maintenant amorcé.}

Au niveau du genou : la concordance morphologique entre les études TDM 3D en décubitus et EOS est bonne, mais l'étude en charge autorisée par le système EOS permet une meilleure étude des déformations qu'elles soient d'origine squelettique, ou des parties ligamentaires et musculaires. Ceci permet déjà de mieux apprécier dans l'espace les déformations au niveau de l'articulation ellemême, mais aussi et surtout de replacer ce genou dans l'ensemble du membre (hanche, genou, pied), mais aussi dans l'ensemble du corps avec en particulier le retentissement d'un côté sur l'autre, de même que le retentissement des altérations morphologiques et fonctionnelles du squelette du tronc.

Au niveau de la hanche : la vision 3D en charge approche de très près la réalité. Le retentissement des éléments sus (bassin, rachis et tronc) et sous jacents (genou, pied) peut être évalué en $3 \mathrm{D}$ ce qui est d'une importance considérable dans l'indication et la réalisation des implantations prothétiques par exemple, de même que pour l'évaluation des résultats.

On connaissait l'étude si difficile des phénomènes et des anomalies de rotations des membres inférieurs où les différentes coupes TDM (hanches [tête et col], condyles fémoraux, extrémité supérieure du tibia, cheville, pied) permettait de faire des mesures utiles pour la pathologie et la thérapeutique, au prix d'une certaine irradiation et d'une étude en position couchée.

Il va de soi que grâce au système EOS, non seulement les mêmes renseignements, mais d'autres encore en particulier sur le plan rotatoire 3D beaucoup plus précis à cause des couplages générés par la position debout, seront découverts.

Tout ceci amènera bien sûr à des études sur la pathologie, les remplacements prothétiques, hanche, genou, et autres, cette fois-ci étudiés dans l'espace et non sur les projections uniplanaires données par les radiographies déjà nettement démarrées au niveau des prothèses de genou.

D'où la création peut-être de nouvelles prothèses, en tout cas de nouveaux critères de contrôle de mise en place primitivement et contrôles de détérioration spatiale à distance.

\section{Contingences actuelles}

L'appareil EOS existe actuellement en deux prototypes, l'un situé en permanence à l'ENSAM à Paris, l'autre qui était à l'hôpital St. Vincent de Paul est actuellement en Belgique, une troisième est en cours d'installation au Canada. 
EOS fonctionne avec un système informatique basé sur PC.

Son installation réclame une salle de radiographies habituelle avec un sol suffisamment stable et renforcé pour supporter le poids de la machine et éviter les vibrations lors du déplacement de ses éléments mobiles au cours de la prise du cliché.

Son coût est de l'ordre de la moitié de celui d'un scanner de dernière génération.

\section{Conclusion}

EOS ouvre un ère nouvelle dans l'orthopédie et l'étude des maladies du squelette en faisant exploser l'importance des trois dimensions de l'espace tout en réduisant significativement les effets possiblement nuisibles d'une irradiation excessive.

\section{RÉFÉRENCES}

Babichev E.A., Baru S.E., Groshev V.R., Khabakhpashev A.G., Leonov V.V., Neustroev V.A., Porosev V.V., Savinov G.A., Shekhtman L.I. (2003) The new effective detector for digital scanning radiography, Nucl. Instrum. Meth. A 513, 57-60.

Berrington de Gonzales A., Darby S. (2004) Risk of cancer from diagnostic X-rays: estimates for the UK and 14 other countries, The Lance 363, 9406.

Deschenes S., Godbout B., Branchaud D., Mitton D., Pomero V., Bleau A., Skalli W., De Guise J.A. (2003) 3D reconstruction of the human spine from Bi-planar radiographs : using multi-scale wavelets analysis a,d splines interpolators for semi-automation, SPIE Medical Imaging.

Kalifa G., Charpak G., Maccia C., Fery-Lemonnier E., Bloch J., Boussard J.M., Attal M., Dubousset J., Adamsbaum C. (1998) Evaluation of a new low-dose digital X-ray device: first dosimetric and clinical results in children, Pediatric Radiology 28, 557-561.

Laporte S., Skalli W., De Guise J.A., Lavaste F., Mitton D. (2003) A biplanar reconstruction method based on 2D and 3D contours: application to the distal femur, Comput. Meth. Biomech. Biomed. Engin. 6, 1-6.

Le Bras A., Laporte S., Mitton D., De Guise J.A., Skalli W. (2003) 3D detailed reconstruction of human vertebrae from dose digital stereoradiography, Eur. J. Orthopaedic Surg. Traumat. 13, 57-62.

Mitton D., Landry C., Veron S., Skalli W., Lavaste F., De Guise J.A. (2000) 3D reconstruction method from biplanar radiography using non stereocorresponding points and elastic deformable meshes, Med. Biolog. Engeer. Comput. 38, 133-139.

Pomero V., Mitton D., Laporte S., De Guise J.A., Skalli W. (2004) Fast accurate stereoradiography 3Dreconstruction of the spine using a combined geometric and statistic model, Clin. Biomech. (Bristol, Avon) 19, 240-247. 